\title{
Artificial dielectric optical structures: A challenge for nanofabrication
}

\author{
C. Giaconia, ${ }^{\text {a) }}$ R. Torrini,, ${ }^{\text {a) }}$ S. K. Murad, and C. D. W. Wilkinson ${ }^{\text {b) }}$ \\ Department of Electronics and Electrical Engineering, Glasgow University, G12 8LT Glasgow, Scotland, \\ United Kingdom
}

\begin{abstract}
(Received 29 May 1998; accepted I September 1998)
Diffractive optical components can be made using multiple level kinoforms or single level artificial dielectric structures. The latter require the fabrication of pillars of equal depth but differing width and spacing. As a demonstration device, the diffractive optic equivalent of a wedge has been made in $\mathrm{GaAs}$ for use at $1.15 \mu \mathrm{m}$. The need for all pillars to have the same height was met by using a selective etch and a very thin etch-stop layer on AlGaAs. The experimental diffraction efficiency was $87.8 \%$, among the best ever obtained and close to the theoretical maximum of $97.6 \%$. (c) 1998 American Vacuum Society. [S0734-211X(98)03706-8]
\end{abstract}

\section{INTRODUCTION}

Diffractive optical components offer, in principle, marked manufacturing advantages in comparison to conventional shaped components, as well as the possibility of performing functions (for example, the generation of multiple spots) which cannot be readily done by conventional components. However diffractive $(0.4$ to $0.7 \mu \mathrm{m})$ optical components, particularly those for use in the visual and at high numerical aperture, present a very significant challenge to the art of nanofabrication. ${ }^{1}$

In this article we discuss how modern electron beam lithography and dry etching techniques can be used to make devices for use in the visual.

There are two main approaches to the realization of high efficiency components in diffractive optics: a multiple level approach to approximate a locally curved profile as closely as possible, and the newer approach (for optics, it is well known in microwave practice) of using artificial dielectrics.

The multiple step approach uses successive steps of pattern definition and etching to create the desired shape. For, say, an approximation of 16 levels $\left(2^{4}\right)$, four pattern definition steps are used and four etching steps; the relative depth of the etching in subsequent steps being $1,2,4$, and 8 . Not only is excellent depth control necessary but each new pattern has to be aligned to the last one, despite the difficulties introduced by the resist profile being more complex on each new etching step.

The artificial dielectrics approach uses features of the same depth but with widths much smaller than the wavelength of the light. The phase of the light is controlled locally by using a series of slots of different widths. These slots are narrow enough to avoid diffraction, thus forming an artificial dielectric. This control of the phase allows the creation of components that can fulfill all the conventional optical requirements.

The great advantage of the approach is that only single level lithography (so, no alignment) is required; the disadvantage is that, in comparison to a multiple level approach,

\footnotetext{
an leave from the Dipartimento di Ingegneria Elettrica, University of Palermo, Palermo, Italy.

${ }^{b}$ Electronic mail: chrisw@elec.gla.ac.uk
}

higher aspect ratios in the features are required.

Artificial dielectric structures can be used to make antireflection elements for the fabrication of beam shaping and beam focusing devices, and for the synthesis of artificially created birefringent materials. ${ }^{2-8}$

Artificial dielectric structures can be designed using a complex theory implemented by several theoretical approaches, such as the rigorous coupled wave approach (RCWA) or the finite element method (FEM) ${ }^{9-13}$ With subwavelength elements, it is necessary to take into account the vectorial nature of an electromagnetic wave.

In this work the design phase was addressed by using a hybrid technique that combines the RCWA and an approximate method called effective medium theory (EMT). ${ }^{2,14}$ The model allows the spatial constraints imposed by fabrication (minimum feature width, maximum aspect ratio) to be included automatically in optimization of the design. ${ }^{15}$

From the manufacturing point of view, a general artificial dielectric is an unequally spaced binary grating. The parameters that have to be controlled are the etch depth which has to be the same in all slots, the position of the transition points which define the slots and grooves of the grating, and the refractive indices of the materials at the working wavelength.

In Sec. II, it will be seen how the difficulties connected with these challenging input constraints were overcome, and in Sec. III the experimental results will be presented.

\section{FABRICATION CONSIDERATION}

\section{A. Outline of the process}

The first device designed was a wedge made of gallium arsenide for operation at a wavelength of $1152 \mathrm{~nm}$. This produces an output beam deflected by $30^{\circ}$ from the initial normally incident beam.

The wedge has an overall period of $2.3 \mu \mathrm{m}$, each divided into seven slots $735 \pm 5 \mathrm{~nm}$ deep, with a minimum pillar width of $50 \mathrm{~nm}$, a minimum slot width of $150 \mathrm{~nm}$, and a maximum aspect ratio of about $14.5: 1$ and $5: 1$ for pillars and grooves, respectively.

To meet these very tight requirements, it proved desirable to have as few steps as possible in the process and, to maxi- 


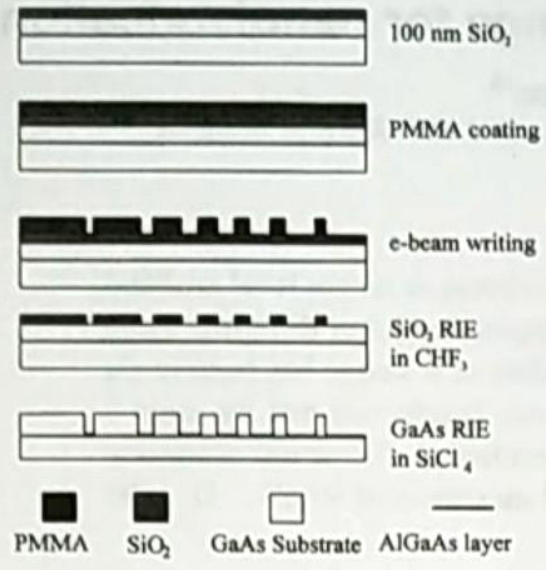

Fio. 1. Schematic of the nanofabrication process.

mize the reliability, to use, as far as possible, established nanolithographic techniques and processes.

It was decided to use the wholly subtractive process shown in Fig. 1. While liftoff of the ion resistant mask is potentially less time consuming, experience showed that is less reliable.

A suitable $\mathrm{GaAs}$ substrate was covered with a $100 \mathrm{~nm}$ thick silicon dioxide layer using a standard plasma enhanced chemical vapor deposition (PECVD) machine. Two layers of PMMA (Elvacite with a molecular weight of 350000 ) diluted with xylene in a $4 \%$ solution was spin coated onto the wafer to give a total thickness of $240 \mathrm{~nm}$. The resist was exposed in a Leica EBPG5-HR100 electron-beam writing machine and developed in 1:3 MIBK:IPA at $23^{\circ} \mathrm{C}$ for $45 \mathrm{~s}$. The silicon dioxide was then reactively ion etched (RIE) in a $20 \mathrm{sccm}$ flow of $\mathrm{CHF}_{3}$ at a total pressure of $15 \mathrm{mTorr}$ and with $100 \mathrm{~W}$ rf power using a process designed to allow the PMMA to be used as a resist while giving a vertical profile. The GaAs substrate was etched in $\mathrm{SiCl}_{4}$ using another RIE process that is described later. Both dry-etching phases were carried out in RIE machines from Oxford Plasma Technology.

\section{B. Lateral resolution issues}

The minimum feature size was $50 \mathrm{~nm}$ which necessitated the use of electron-beam (e-beam) lithography to define the master pattern. The e-beam machine used in this work has a spot size that is adjustable from $10 \mathrm{~nm}$ upwards and a minimum beam deflection step of $5 \mathrm{~nm}$. The overall field size depends on the deflection step. The stitching error between fields is of the order of $80 \mathrm{~nm}$. A suitable compromise between the conflicting aims of high spatial fidelity that would give the correct local phase and a large field was found to be a $28 \mathrm{~nm}$ spot size and a $12.5 \mathrm{~nm}$ deflection step which allowed a $400 \times 400 \mu \mathrm{m}$ and confined the device inside one field. With these parameters no proximity correction was necessary at $50 \mathrm{keV}$ of acceleration voltage.

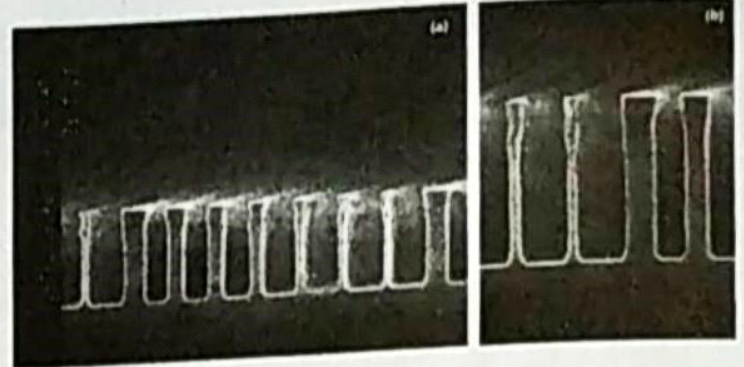

FIG. 2. Scanning electron micrographs of (a) one main period of the artificial dielectric $(2.3 \mu \mathrm{m})$ and $(\mathrm{b})$ a detail showing smallest pillar and groove. dielectric $(2.3 \mu \mathrm{m})$ and (b) a detail showing smallest pillar and groove.

\section{Pattern transfer considerations}

When deep slots are made by reactive ion etching, it is often found that the etch depth varies markedly from feature to feature depending on its width. The variation in depth, after the same etch time, for features varying in aspect ratio from $1: 1$ to $10: 1$ can be as much as $2: 1$. This phenomenon is called RIE lag. Proper realization of the present device requires a uniform etch depth. To overcome this very real difficulty an etch stop and a highly selective etching process which does not etch the stop level were used.

So, a $90 \mathrm{~nm}$ thick $\mathrm{Al}_{0.4} \mathrm{Ga}_{0.6}$ As stop layer was deposited by molecular beam epitaxy (MBE) onto the GaAs wafer before the $735 \mathrm{~nm}$ thick GaAs layer that is designed to give the correct phase shift. The aluminum in the layer underneath was chosen to be sufficient to give very high selectivity in the etching process. Its presence does introduces a constant phase shift since the refractive index of AlGaAs differs from that of GaAs.

The wafer was then used to carry out the processing, as shown in Fig. 1. In the last etching phase, the pattern was transferred into the GaAs using silica dioxide as a mask. The sample was reactively ion etched in silicon tetrachloride with $15 \mathrm{~W}$ rf power, a $3 \mathrm{sscm}$ flow giving a $4 \mathrm{mT}$ Torr of total pressure inside the chamber, and a bias of around $100 \mathrm{~V}$.

These low pressure and low power conditions give vertical sidewalls, while realizing a selective process which stops on the AlGaAs layer. ${ }^{16} \mathrm{~A}$ slightly higher starting pressure in the chamber is sometimes necessary to minimize the induction time of the etching process. This leads to the wiggle at the top of the structures seen in Fig. 2.

The etch rate in open areas, say areas more than a few microns wide, was about $50 \mathrm{~nm} / \mathrm{min}$ so the AlGaAs layer was reached in around $15 \mathrm{~min}$. The etching process assured enough $\mathrm{GaAs} / \mathrm{AlGaAs}$ selectivity to permit an amazing 30 min of overetching which insured uniform depth in all the slots. Finally, the oxide layer was removed by wet etching in a buffered solution of HF prior to testing.

\section{Design considerations}

As we saw in Sec. II C, a lot of effort was expended in order to find a reliable fabrication process, taking into account the diffractive requirements. On the other hand, the 
fabrication process imposed some technological limits but they were used in the design phase to find the best link between design and fabrication issues.

First of all, the finite dimension of the realizable features, dictated by the physical resolution limits of the fabrication process, allowed a maximum theoretical diffraction effi-
ciency of $97.6 \%$.

Among these satisfactory solutions, the best set of parameters chosen (transition points and depth of the grating) were the ones with transition points spaced at multiples of the e-beam deflection step size selected. In addition, a subsequent filtering action chose the solutions with the lowest sensitivity against fabrication errors in the location of the transition points. Finally, the different refractive index of $\mathrm{AlGaAs}$ was also incorporated into the design phase through the proper choice of its thickness.

\section{EXPERIMENTAL RESULTS}

The results obtained can be analyzed from the micrographs shown in Figs. 2(a) and 2(b). From the lateral resolution point of view, the tolerance between the pattern designed and the real device was kept within one step of the resolution grid, that is, $12.5 \mathrm{~nm}$.

A very uniform etch depth can clearly be seen even in the region with the biggest change in aspect ratio. The etching process gives very vertical features; the thinnest pillars are less than 200 atoms wide, and have aspect ratios well above $10: 1$.

For the optical point of view, the diffraction efficiency measurements were carried out by positioning the grating in front of a $75 \mu \mathrm{m}$ spot from a near-infrared helium-neon laser working at a $1152 \mathrm{~nm}$ wavelength. Only three propagating orders were present, as is easily deduced from the designed periodicity, and the experimental diffraction efficiency of the first transmitted order was $87.8 \%$ of the total power transmitted. During the measurements the samples were always coated with a PMMA layer that acted as an antireflection coating. The PMMA layer does not have the correct refractive index to match GaAs perfectly with air, and this may explain the difference between the diffraction efficiency that resulted from the design and that obtained experimentally. However this diffraction efficiency value is, to the best of the authors' knowledge, among the best seen so far.

\section{ACKNOWLEDGMENTS}

The authors wish to thank all the Department of Electronics technical staff for the continuous help given in the fabrication process. A special thanks to Adam Boyd for supplying the MBE grown wafers used during the experiments. One of the authors (C.G.) wants to thanks the European Community who partially financed his research under the Training and Mobility of Researcher (TMR) Program.

'C. Amone, C. Giaconia, and G. Lullo, Diffractive Optic and Optical Microsystem, edited by S. Martellucci et al. (Plenum, New York, 1997), pp. 119-131.

${ }^{2}$ D. H. Raguin and G. M. Morris, Laser Focus World 33, 113 (1997).

${ }^{3}$ J. R. Wendt, G. A. Vawter, R. E. Smith, and M. E. Warren, J. Vac. Sci. Technol. B 13, 2705 (1995).

4J. R. Wendt, G. A. Vawter, R. E. Smith, and M. E. Warren, J. Vac. Sci Technol. B 15, 2946 (1997).

${ }^{5}$ F. T. Chen and H. G. Craighead, Opt. Lett. 20, 177 (1996)

${ }^{6} \mathrm{~S}$. Babin and A. Tomnikov, Microelectron. Eng. 27, 167 (1995).

${ }^{7}$ F. Xu, R. C. Tyan, P. C. Sun, Y. Fainman, C. C. Cheng, and A. Scherer, Opt. Lett. 20, 2457 (1995).

${ }^{8}$ W. Parkes, S. Thoms, and C. D. W. Wilkinson, Microelectron. Eng. 23, 465 (1994).

${ }^{9}$ K. Knop, J. Opt. Soc. Am. 68, 1206 (1978).

${ }^{10} \mathrm{~T}$. K. Gaylord and G. Moharam, Proc. IEEE 73, 894 (1985).

"W. B. Veldkamp, G. J. Swanson, S. A. Gaither, C. L. Chen, and T. R. Osborne, MIT Report ODT 20, 1989.

${ }^{12}$ T. Delort and D. Maystre, J. Opt. Soc. Am. 10, 2592 (1993).

${ }^{13}$ M. K. Moaveni, Proc, IEE 126, 35 (1979).

${ }^{14}$ S. M. Rytov, Sov. Phys. JETP 2, 466 (1956).

${ }^{15} \mathrm{C}$. Giaconia, R. Torrini, S. Murad, and C. D. W. Wilkinson, Digest of Diffractive Optics and MicroOptics, OSA Summer Topical Meetings, Hawaii, 1998 (to be published).

${ }^{16}$ S. K. Murad, C. D. Wilkinson, and S. P. Beaumont, Microelectron. Eng. 23, 357 (1994) 
Wแด

B6rarbe

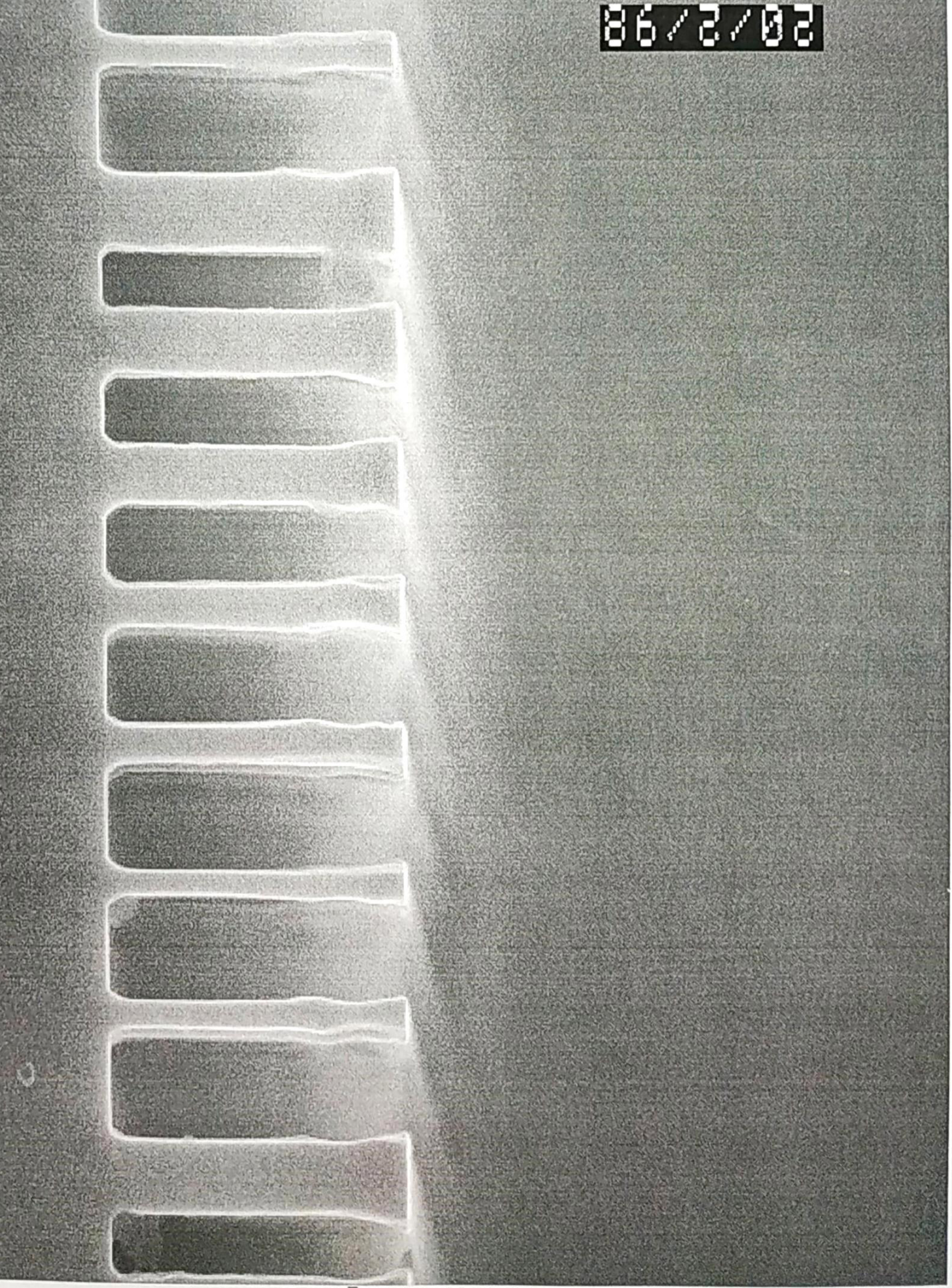

